\title{
Effects of Stress Induced by Preventing the Thermal Contraction on the Amount of Retained Austenite*
}

\author{
By Iwao Hagiwara, ** Shogo Kanazawa** and Uko Kumada**
}

\begin{abstract}
Thermal contraction while cooling was prevented as a representation of a special thermal stress in the surface layer of sieel induced by quenching. The effects of such thermal stress on retained austenite $\left(\gamma_{R}\right)$ were as follows. (A) Experiments on high carbon alloy steels. (1) The tensile stress induced by preventing the thermal contraction increased linearly with decreasing temperature and indicated the maximum value at $\mathrm{Ms}$ temperature, that was about $10 \mathrm{~kg} / \mathrm{mm}^{2}$. (2) The amount of $\gamma_{\mathrm{R}}$ was markedly increased by preventing the thermal contraction. This is contrary to the expectation from the mechanism of martensite transformation which has been interpreted from the criterion of shearing stress. (3) The effective temperature range of stress on $\gamma_{R}$ existed between $100 \sim 150^{\circ} \mathrm{C}$ above Ms point and room temperature. (4) The rise of cooling speed at the range of above Ms temperature increased the a mount of $\gamma_{R}$ as the result of increment of induced tensile stress. (5) Below the Ms temperature, the rise of cooling speed decreased the amount of $\gamma_{R}$, similarly to free contraction cooling. Therefore it appears that there is no offect of stress on the thermal stabilization of austenite. (B) Stabilization of austenite by preventing thermal contraction was not observed in medium and low carbon alloy steels, and in low carbon alloy steel the amount of $\gamma_{R}$ was decreased, contrary to the case with high carbon alloy steels.
\end{abstract}

(Received September 5, 1930)

\section{Introduction}

Under certain loading Fe-Ni alloys undergo a cooling transformation. The martensite (later abbreviated to $M$ ) transformation is increased.(1)(2) This phenomena can be explained by the fact that $M$ transformation belongs to plastic deformation like mechanical slip or twin deformation, and that the driving force of the transformation is the difference of free energy of austenite (later abbreviated to $\gamma$ ) and martensite.

It can be explained that the shearing stress applied from the outside increases the drivnig force and promotes $M$ transformation. Such an effect of stress shall be called the "Shearing Stress Effect". As the other example of the stress effect applied from outside, there is an effect of normal stress on the work done of transformig expansion, and this shall be called "Normal Stress Effect". This normal stress effect, when worked as tension, has an agency to promote transformation, and when worked as compression, it turns out to be a preventing force. But compared with the shearing stress effect, its influence is comparatively small, and the resultant force of these two stress effects are always promoting, though the tensile stress is calculated to be of stronger effect. ${ }^{(4)(5)}$

** Faculty of Technology, Hokkaido University.

* This paper was published in originally in Japanese in the Journul of the Japan Institute of Metals, 23 (1959), 299.

(1) E. Sheil : Z. anorg. allg. Chem., 207 (1932), 21.

(2) A. W. Mc Rynolds: J. Appl. Phys., 20 (1949), 896.

(3) B.S. Lement, B. L. Averbach and M. Cohen: ASM, 47 (1955), 217.

(4) J. C. Fisher, D. Turnbull : Acta Met., 1 (1953), 310.

(5) J.R. Patel, M. Cohen: Acta Met., 1 (1953), 531.
The stress produced in the quenching of steel makes complex changes according to quenching conditions. For instance we shall take up the stress of the surface layer which is subject to rapid cooling. Owing to the prevention of free contraction, the tensile stress increases with the fall of the surface temperature, presumably attaining to the maximum at the Ms point. Below the Ms point, the differences of thermal contraction and transforming expansion outside and inside have complex effects on each other. The decrease of tensile stress may sometimes be succeeded by the increase of the same stress, or in some cases it may be followed by the appearance of a compressive stress. In presuming such changes, the results of measurement about residual stress in quenching may be available.

Whatever may be the change of the stress below the Ms point, the retained austenite (later abbreviated to $\gamma_{\mathrm{R}}$ ) should decrease by the stress induced by quenching. Because, as we stated before, $M$ transformation is thought to be promoted by either tensile stress or compressive stress. The idea that quenching stress will generally decrease $\gamma_{\mathbf{R}}$ is not a new one.(6) It has been counted as a cause of the difference of $\gamma_{R}$ amount between oil quenching and water quenching. It has been taken for granted and therefore has not been fully investigated.

As a representation of a special case of thermal stress in the surface layer induced by quenching of high carbon alloy steels, we have made experiments on the effect of thermal stress induced by preventing the contraction in the axial direction of the cooling specimen on the amount of $\gamma_{\mathrm{R}}$. The experimental

(6) K. Honda, K. Iwase : Research of M 3, (1926), 326. 
result was contrary to the expectation from the criterion of shearing stress, because the amount of $\gamma_{\mathrm{R}}$ was increased by preventing the thermal contraction.

In this first report we are going to present the results of the experiment. And as we think that this contains not only a problem of heat treatment, but also a problem concerning the mechanism of transformation we are going to make further reports about the results of different experiments on the influence of stress.

\section{Test Specimens and the Method of Experiments}

The test specimen is a bar $5 \mathrm{~mm}$ in diameter and $100 \mathrm{~mm}$ in length with a $10 \mathrm{~mm}$ screw cut on both ends and nuts attached. For the prevention of thermal contraction, the apparatus shown in Fig. 1 was used. The test specimen that has been

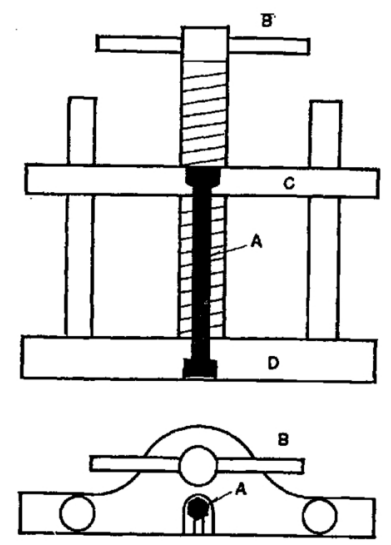

Fig. 1 Apparatus used for the experiments.

austenitized at $950^{\circ} \mathrm{C}$ is quickly set in the apparatus as $A$. When the specimen has been cooled down to the prescribed temperature, the handle $B$ is turned and the heads of both nuts attached close to bases $C$ and D. For the measurement of the temperature of the specimen, a thermo couple was attached to the middle part of the specimen. For the measurement of cooling speed, a micro-recorder (high speed self-recording thermometer) was used. For the measurement of amount of $\gamma_{R}$, the differential dilatation test in tempering, magnetic analysis, and microscopic test were made on $80 \mathrm{~mm}$-long bars with the screw ends cut off. The differential dilatation (heating speed is $2^{\circ} \mathrm{C} / \mathrm{sec}$ ) was employed comparing the amount of extraordinary expansion induced by the decomposition of $\gamma_{R}$, using the neutral bar that is completely annealed steel as the same kind of specimen. Also magnetic analysis is employed by the same method. The microscopic test was done by point counting the several microscopic photographs of the cross section excepting the centre and the outermost parts.

Fig. 2 shows that the three methods agreed very well so in the subsequent experiment the differential dilatation tests were chieply used. As test material, we used two kinds of high carbon alloy steels, namely Cr steel (steel A) which is used as bearing and hard surface roll, and Ni steel (steel B) which has often been used in many laboratories for the study of stabilization of $\gamma$ (Table 1). About both steels we affirmed that the $\mathrm{Ar}^{\prime}$ transformation was completely

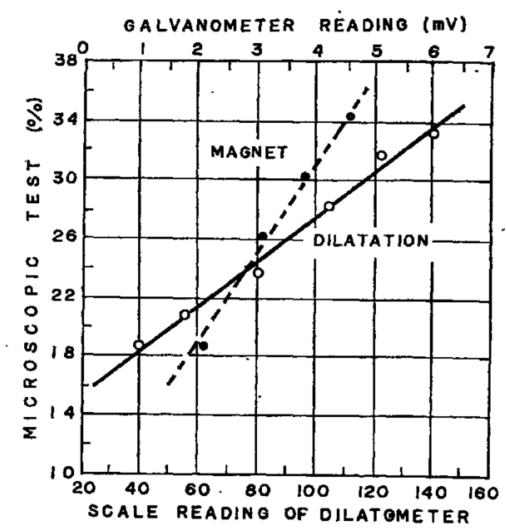

Fig. 2 Relation of retained austenite obtained by mictoscopic, magnetic and dilatometric methods.

Table 1 Composition of specimens.

\begin{tabular}{c|c|c|c|c|c|c|c}
\hline Elements & $\mathrm{C}$ & $\mathrm{Si}$ & $\mathrm{Mn}$ & $\mathrm{P}$ & $\mathrm{S}$ & $\mathrm{Ni}$ & $\mathrm{Cr}$ \\
\hline Steel & & & & & & & \\
\hline $\mathrm{A}$ & 0.99 & 0.25 & 0.64 & 0.021 & 0.029 & 0.08 & 2.86 \\
$\mathrm{~B}$ & 1.00 & 0.23 & 0.51 & 0.018 & 0.024 & 4.99 & 0.05 \\
\hline
\end{tabular}

prevented by cooling in air. Therefore the quenching was done chiefly in air. We also made tests using medium carbon and low carbon alloy steel, the composition of which will be introduced later.

\section{The Results of Experiments and the Examination}

(a) The stress induced by prevention of contraction

As it was difficult to measure the stress with the apparatus in Fig. 1, an Amsler tester was used for this purpose. A specimen heated up to $950^{\circ} \mathrm{C}$ was quickly ( 1 second) put in the $500 \mathrm{~kg}$ Amsler tester and immediately the first load of $5 \mathrm{~kg}$ (about $0.2 \mathrm{~kg}$ )

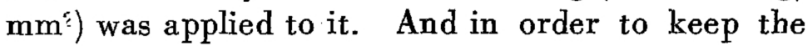
specimen the same length through out the cooling process, the load was applied continuously so that the stress-strain diagram on the rotating drum may be a complete straight line parallel to the spindle. Experiments were repeated several times on each steel. And judging from the accuracy of the lines, the error was not larger than $\pm 2 \sim 3 \%$. Fig. 3, is the results of the test in which the specimen was cooled in air from $930^{\circ} \mathrm{C}$, the contraction being prevented by the above mentioned method. With the fall of the temperature, the stress increases in straight lines, reaching the maximum at the $\mathrm{Ms}_{\mathrm{s}}$ point, below which temperature the stress abruptly decreases owing to the dilatation induced by transformation, but at room temperature the tensile stress still remains. We obtained the stress at room temperature by calculating the difference of length before and after the specimens were taken from the tester, and with the reported elastic modulus of $M$ 
$\left(2.05 \times 10^{4} \mathrm{~kg} / \mathrm{mm}^{2}\right)$.(7) The change of stress from the Ms temperature down to room temperature was obtained by presuming from the transformal dilatation curve.

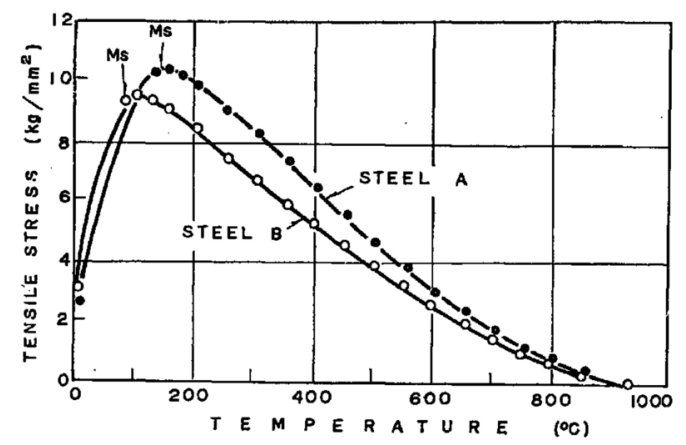

Fig. 3 Tensile stress induced by preventing the thermal contraction from $930^{\circ}$ to room temperature.

(b) The influence of prevention of contraction

After having completely austenitized the A and B steel by keeping the former for $50 \mathrm{~min}$ and the latter for $30 \mathrm{~min}$ at $950^{\circ} \mathrm{C}$, we studied the change of the amount of $\gamma_{R}$ induced by preventing contraction from various temperture in the course of air-cooling down to room temperature. The results are shown in Fig. 4. Both kinds of steel show the same change. The higher the temperature at which the contraction is prevented, namely the larger the thermal stress, the more marked is the increase of $\gamma_{R}$, and above $800^{\circ} \mathrm{C}$ it is twice as large as in the case of free contraction. When in some parts $\mathrm{Ar}^{\prime}$ transformation take place, the $\gamma$ of the rest is sometimes stabilized,(8)(9) and at the same time when stress is applied, $\mathrm{Ar}^{\prime}$ transformation tends to be promoted.(10) But in this experiment it was confirmed that in spite of the increase of stress no $\mathrm{Ar}^{\prime}$ transformation had occurred.

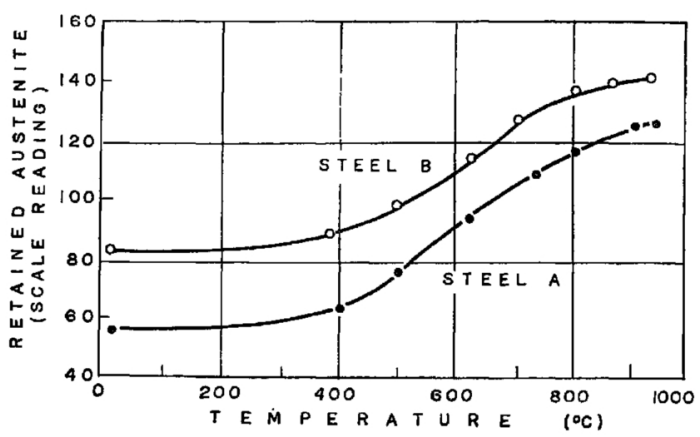

Fig. 4 Retained austenite obtained by preventing the thermal contraction from various temperatures.

We air-cooled the steels from $930^{\circ} \mathrm{C}$ under the prevention of contraction, and released the prevention at various temperature and then let them cooled in the free state down to room temperature. The results are shown in Fig. 5. According to these results, $\gamma_{\mathrm{R}}$ increases markedly in the temperature range around the Ms point (measured in free state) and in

(7) Ueda, Kumei : J. Japan Inst. of M., 17 (1953), 623.

(8) S. S. Steinberg: Arch. Eisenhutt., 7, (1934), 537.

(9) U. Imai: Japan Inst. of M., 7 Report 11 (1947), 9.

(10) I. Hagiwara, S. Kanazawa, U.Kumada: Committee of Japan Inst. of M., 1956. the other temperature ranges it makes little change. As the cause of this sudden increase around the Ms point cannot be observed in the change of thermal stress (Fig. 3), only the stress in a certain temperature

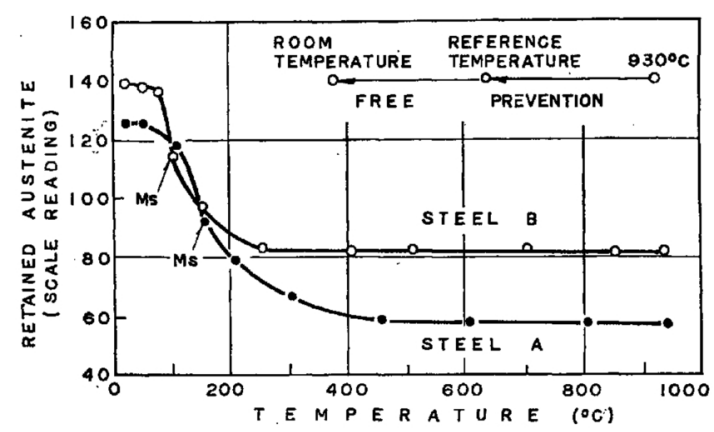

Fig. 5 The effective temperalure range of stress on retained austenite.

range around the Ms point is presumably effective for the stabilization of $\gamma$. Such stabilization of $\gamma$ is a contrary phenomenon to the stress effect mentioned in the introduction.

Thus a certain relation can be expected between $\gamma_{R}$ and the stress around the Ms point. It may be given as follows. The relation between the temperature at which the prevention of contraction begins $\left(\theta_{i}\right)$ and the stress at Ms point $\left(\sigma_{m}\right)$ can be obtained from the curve in Fig. 3 . Let the curve be $\sigma=f(\theta)$, and $\sigma_{m}$ is approximately equal to $\mathrm{f}(\mathrm{Ms})$ $\mathrm{f}\left(\theta_{i}\right)$. We can get $\Delta \gamma_{\mathrm{R}}$ (the difference of $\gamma_{\mathrm{R}}$ between the free state and under prevention of contraction) from Fig. 4. The relation between $\Delta \gamma_{\mathbf{R}}$ and $\sigma_{m}$ is shown in Fig. 6. There is a slight difference between $A$ and $B$ steels, but they are of almost the same curve. In our next report, it shall be shown that this curve nearly coincides with the results

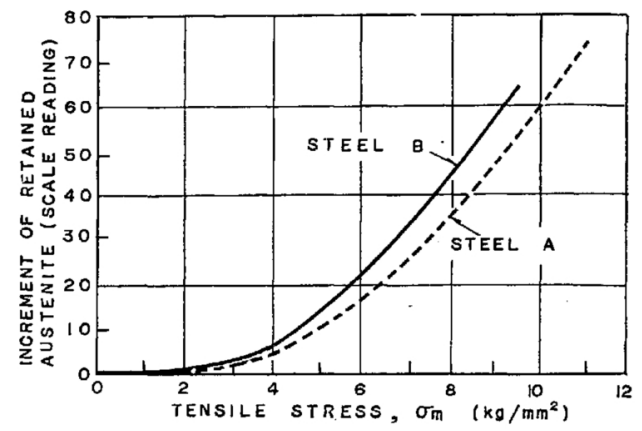

Fig. 6 Relation between tensile stress at Ms temperature and the increment of retained austenite.

obtained by applied loading.

The increase of $\gamma_{R}$ is given even when the contraction is prevented only above the Ms temperature as is shown in Fig. 5. This fact and the relation between stress and $\gamma_{\mathrm{R}}$ in Fig. 6 may suggest that the stabilization of $\gamma$ is due to work hardening. But there is other experiments in which work promote transformation.(1)(3) So the conclusions will be presented in the next report. And even if the work hardening is its cause, the question why the stress gives work hardening without promoting transformation will remain unanswered. 


\section{(c) The influence of cooling speed}

As a factor of stabilization of $\gamma$, there is thermal stabilization besides mechanical stabilization like work hardening. That $\gamma_{\mathrm{R}}$ decreases with the increase of cooling speed is one example. The relaxation of transformation stress, strain age hardening, the decrease of embryos of $M$, and many others are considered to have some relation to the thermal stabilization. In either case, $\gamma$ which is deformed by stress is likely to be promoted in thermal stability. If so the stabilization of stress should in marked degree be decreased by rapid cooling. Fig. 7 shows

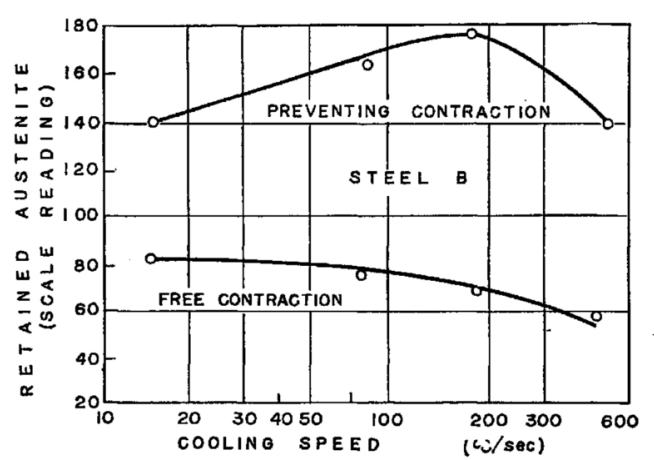

Fig. 7 Effect of cooling speed (mean of $850 \sim 600^{\circ} \mathrm{C}$ ) under preventing and free contraction.

the effect of cooling speed. One is a case in which the specimen was cooled from $950^{\circ} \mathrm{C}$ to room temperature in the free condition, and the other is cooled under prevention of contraction (the cooling speed in Fig. 7 is the mean of $850^{\circ} \sim 600^{\circ} \mathrm{C}$ ). In the free state, with the increase of the quenching cooling speed, $\gamma_{R}$ decreased. This is, as is already known, (11) due to the thermal stabilization that occurs below $\mathrm{Ms}$ (more accurately $\sigma s$ ). While under prevention of contraction, contrary to the expectation, with the increase of cooling velocity $\gamma_{R}$ increases, attaining the maximum and later passing into a decreasing.

Next another test was made by cooling the specimen in boiling water in order to keep the cooling

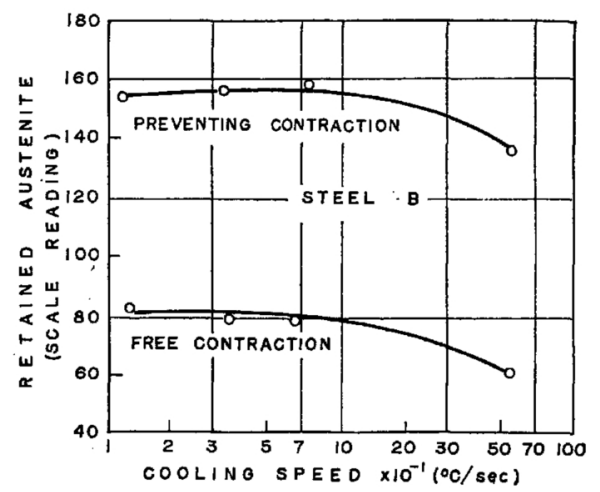

Fig. 8 Effect of cooling speed below Ms temperature, under preventing and free contraction.

condition constant from $950^{\circ} \mathrm{C}$ to $\mathrm{Ms}$ temperature $\left(105^{\circ} \mathrm{C}\right)$, and later changing speed only below the Ms temperature. The results are shown in Fig. 8.

(11) W. J. Harris, M. Cohen : Metal Technol. T. P. 2246. Sept. (1948).
Under prevention of contraction $\gamma_{R}$ was observed to decrease with the increase of cooling speed as in the free state. Therefore the increase of $\gamma_{R}$ with the increase of cooling speed under prevention of contraction is effected by the cooling speed above the Ms temperature. This may be attributed to the increase of tensile stress induced by the increase of cooling speed. Further, the decrease of $\gamma_{R}$ after having attained maximum at a certain cooling speed may be due to the opposed effect by the increase of the cooling speed below the Ms temperature. After all the results of the above mentioned experiments show that stress has little effect on thermal stabilization of $\gamma$, which leads to a conclusion that the stabilization by tensile stress is not due to the thermal stabilization.

\section{(d) The effect of carbon content of steels}

For comparison we made a test of prevention of contraction about the medium carbon alloy steel (steel C) and the low carbon alloy steel (steel D) as shown in Table 2. Both are of hardenability that makes complete hardening possible by air-cooling. The results of measurement of $\mathrm{Ms}$ and $\mathrm{Mf}$ are shown in Table 3. The results of the test with the medium carbon alloy steel shows that no matter at what temperature the prevention of contraction may begin, the results are the same as in the case of free contraction. As to low carbon alloy steel, the amount of $\gamma_{\mathrm{R}}$ is generally very small. But the higher the temperature at which the prevention of contraction begins, the more the decrese of $\gamma_{R}$ is discernible, and the effect of stress is observed to occur in the ordinary way. Table 3 shows two cases, one in which the prevention begins at $930^{\circ} \mathrm{C}$ and one of free contraction. It is noteworthy that the effect of contractive stress should show the reverse tendency to $\gamma_{\mathrm{R}}$ with the decrease of carbon content.

Table 2 Composition of specimens.

\begin{tabular}{c|c|c|c|c|c|c|c|c}
\hline \hline Element & C & Si & Mn & P & S & Ni & Cr & Mo \\
\hline Steel & & & & & & & & \\
\hline C & 0.58 & 0.41 & 0.64 & 0.025 & 0.031 & 4.94 & 0.06 & tr \\
D & 0.19 & 0.40 & $\mathbf{0 . 6 2}$ & 0.026 & 0.028 & 0.07 & 5.46 & $\mathbf{0 . 4 5}$ \\
\hline
\end{tabular}

Table 3 Effects of stress on medium and low carbon alloy steel.

\begin{tabular}{c|c|c|c|c}
\hline \multirow{2}{*}{ Steel } & \multicolumn{2}{|c|}{$\begin{array}{c}\text { Retained austenite } \\
\text { (Scale roading) }\end{array}$} & Ms & Mf \\
\cline { 2 - 4 } & free & prevention & & \\
\hline C & 32 & 32 & $220^{\circ}$ & $45^{\circ}$ \\
D & 7 & 6 & $405^{\circ}$ & $325^{\circ}$ \\
\hline
\end{tabular}

\section{Summary}

As a representation of a special case of thermal stress in the surface layer induced by quenching, a series of experiments was made about the effect of stress induced by preventing the thermal contraction on the amount of retained austenite $\left(\gamma_{\mathrm{R}}\right)$.

(A) In the case of high carbon alloy steel 
(1) The tensile stress induced by the prevention of contraction attains to maximum around $\mathrm{Ms}$ temperature and it was about $10 \mathrm{~kg} / \mathrm{mm}^{2}$.

(2). $\gamma_{R}$ increases remarkably by the prevention of contraction. This phenomenon is found to be contrary to what is expected from the shearing stress theory about martensite transformation that has hitherto been accepted.

(3) The temperature range in which the stress has effect on the amount of $\gamma_{R}$ under prevention of contraction is from $100^{\circ} \sim 150^{\circ} \mathrm{C}$ above the $\mathrm{Ms}$ temperature to room temperature.

(4) In the case of prevention of contraction, above the Ms temperature, the increase of the cooling speed promotes $\gamma_{R}$ as a result of the increase of tensile stress.
(5) The increase of cooling speed below the Ms temperature causes the decrease of $\gamma_{R}$, both in the case of prevention of contraction and of free contraction. Therefore it may be recognized that the stress has little effect on the thermal stabilization of austenite $(\gamma)$.

(B) In the case of medium carbon alloy steel, the stabilization of $\gamma$ is not induced by preventing contraction. Reversely the decrease of $\gamma_{R}$ was discernible with the low cabon alloy steel. Namey the low carbon alloy steel and high carbon one presented just the reverse phenomena about the amount of $\gamma_{\mathrm{R}}$.

Lastly we express our heartfelt thank to $\mathrm{Mr}$. Kaneko who was kind enough to assist us in this experiment. 